\title{
Combinatorial properties of generalized hypercube graphs
}

\author{
Dyi-Rong Duh ${ }^{*}$,a , Gen-Huey Chen ${ }^{a}$, D. Frank Hsu ${ }^{b}$ \\ ${ }^{a}$ Department of Computer Science and Information Engineering, National Taiwan University, Taipei, Taiwan \\ ${ }^{\mathrm{b}}$ Department of Computer and Information Science, John Mulcahy Hall 340, Fordham University, Bronx, NY 10458, USA \\ Received 7 April 1995; revised 9 October 1995 \\ Communicated by M.J. Atallah
}

\begin{abstract}
This paper investigates combinatorial properties of generalized hypercube graphs including best containers, wide diameter, and fault diameter. These properties have received much attention recently in the study of interconnection networks.
\end{abstract}

Keywords: Algorithms; Graph theory

\section{Introduction}

Generalized hypercube graphs are the underlying graphs of generalized hypercube networks [1] which were proposed for building massively parallel computer systems. Let $G\left(m_{r}, m_{r-1}, \ldots, m_{1}\right)$ denote a generalized hypercube graph of size $m_{r} \times m_{r-1} \times \cdots \times m_{1}$, where $m_{i} \geqslant 2$ for all $1 \leqslant i$ $\leqslant r$. There are $N=m_{r} * m_{r-1} * \cdots * m_{1}$ nodes in $G\left(m_{r}, m_{r-1}, \ldots, m_{1}\right)$ which are assigned $r$-digit identifiers $x_{r}, x_{r-1} \ldots x_{1}$, where $x_{i} \in\left[0, m_{i}-1\right]$ for all $1 \leqslant i \leqslant r$. Two nodes in $G\left(m_{r}, m_{r-1}, \ldots\right.$, $m_{1}$ ) arc adjacent if and only if their identifiers differ at exactly one digit position. In Fig. 1, the structure of $G(4,3,2)$ is depicted for illustration.

\footnotetext{
* Corresponding author. Email: ghchen@csie.ntu.edu.tw
}

Each node in $G\left(m_{r}, m_{r-1}, \ldots, m_{1}\right)$ has degree $\sum_{i=1 \ldots r}\left(m_{i}-1\right)$.

In this paper, several combinatorial properties of $G\left(m_{r}, m_{r-1}, \ldots, m_{1}\right)$ are investigated. Specifically, best containers of width $\kappa$, the $\kappa$-wide diameter, and the fault diameter of $G\left(m_{r}, m_{r-1}\right.$, $\left.\ldots, m_{1}\right)$ are computed, where $\kappa$ is the node connectivity of $G\left(m_{r}, m_{r-1}, \ldots, m_{1}\right)$. These properties have become more and more important recently in the study of reliability, fault tolerance, randomized routing, and transmission delay in interconnection networks $[3,5,6]$. It is of both theoretical interest and practical importance to determine a container of width $\kappa$ between arbitrary two nodes because the existence of such a container means that messages can be transmitted in parallel using $\kappa$ disjoint paths. Besides, the transmission will succeed even if $\kappa-1$ node faults have occurred. The fault diameter, on the other hand, estimates the maximum transmission delay under the situation of at most $\kappa-1$ node faults. 


\section{Containers}

Let $X, Y$ be two arbitrary nodes in $G\left(m_{r}, m_{r}, \ldots, m_{1}\right)$. An $(X, Y)$-container [5], denoted by $C(X, Y)$, is a set of node-disjoint paths between $X$ and $Y$. The width of a $C(X, Y)$, denoted by $w(C(X, Y))$, is its cardinality. The length of a $C(X, Y)$, denoted by $l(C(X, Y))$, is defined as the maximum length of the paths in the $C(X, Y)$. A $C(X, Y)$ is the best, denoted by $C^{*}(X, Y)$, if its length is minimum among those with the same width.

Let $X=x_{r} x_{r-1} \ldots x_{1}$ and $Y=y_{r} y_{r-1} \ldots y_{1}$, be two arbitrary nodes in $G\left(m_{r}, m_{r-1}, \ldots, m_{1}\right)$. A particular $C(X, Y)$ of width $\sum_{i=1 \ldots r}\left(m_{i}-1\right)$ has been found in [1]. In this section, a general approach for constructing $C(X, Y) \mathrm{s}$ of width $\sum_{i=1 \ldots r}\left(m_{i}-1\right)$ is first proposed. The constructed paths fall into three sets $S_{1}, S_{2}$ and $S_{3}$ according to their lengths.

Assume $X$ and $Y$ differ at $d$ digit positions: $s(d), s(d-1), \ldots, s(1)$. That is, $x_{i}=y_{i}$ if and only if $i \notin\{s(d), s(d-1), \ldots, s(1)\}$. We let

$$
\begin{aligned}
S_{1}= & \left\{\left(X, X_{s(1)}, X_{s(1), s(2)}, \ldots, X_{s(1), s(2) \ldots \ldots s(d)}=Y\right),\right. \\
& \left(X, X_{s(2)}, X_{s(2), s(3)}, \ldots,\right. \\
& \left.X_{s(2), s(3), \ldots, s(d), s(1)}=Y\right) \\
& \vdots \\
& \left(X, X_{s(d)}, X_{s(d), s(1)}, \ldots,\right. \\
& \left.\left.X_{s(d), s(1), \ldots, s(d-1)}=Y\right)\right\}
\end{aligned}
$$

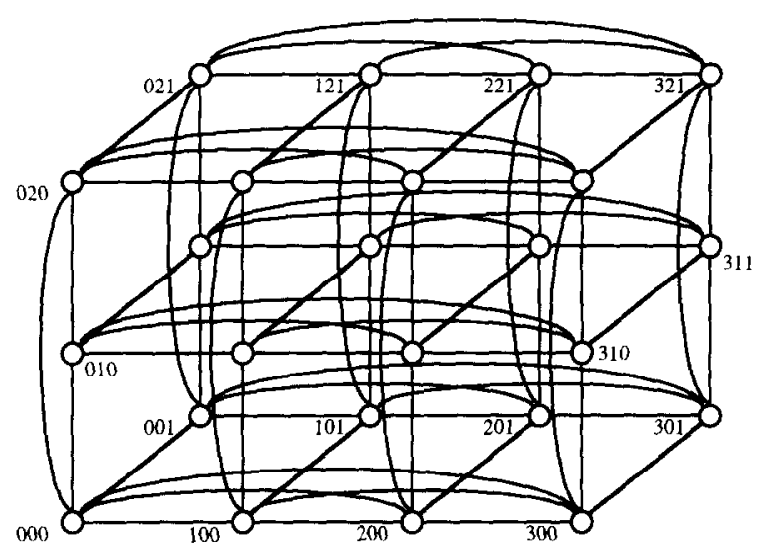

Fig. 1. The structure of $G(4,3,2)$. where $X_{s(i), s(j), \ldots, s(l)}$ represents the identifier resulting from $X$ after replacing $x_{s(i)}, x_{s(j)}, \ldots, x_{s(l)}$ with $y_{s(i)}, y_{s(j)}, \ldots, y_{s(l)}$, respectively.

The set $S_{1}$ contains $d$ paths whose length is $d$.

Further, let $X_{(k, a), s(i), s(j), \ldots, s(l)}$ represent the identifier which is obtained by replacing the $k$ th digit from the right of $X_{s(i), s(j), \ldots, s(l)}$ with $a$. We let

$S_{2}=\left\{S_{2,1}, S_{2,2}, \ldots, S_{2, d}\right\}$,

where for $i=1, \ldots, d$,

$$
\begin{aligned}
S_{2, i}= & \left(X, X_{(s(i), j)}, X_{(s(i), j), s(1)}, X_{(s(i), j), s(1), s(2)}, \ldots,\right. \\
& X_{(s(i,) j), s(1), s(2), \ldots, s(i-1)}, \\
& X_{(s(i), j), s(1), s(2), \ldots, s(i-1), s(i+1)}, \ldots, \\
& X_{(s(i), j), s(1), s(2), \ldots, s(i-1), s(i+1), \ldots, s(d)}, \\
& \left.X_{s(1), s(2), \ldots, s(d)}=Y\right) \mid \text { for } j=0, \ldots, m_{i}-1 \\
& \text { and } \left.j \neq x_{s(i)}, j \neq y_{s(i)}\right\} .
\end{aligned}
$$

If $m_{i}=2$, then no legal $j$ can be found, in which the set $S_{2, i}$ is set to empty. The set $S_{2}$ contains $\sum_{i=1 \ldots d}\left(m_{s(i)}-2\right)$ paths whose length is $d+1$.

Assume $t(r-d), t(r-d-1), \ldots, t(1)$ are the $r-d$ digit positions such that $x_{i}=y_{i}$ if and only if $i \in\{t(r-d), t(r-d-1), \ldots, t(1)\}$. We let

$S_{3}=\left\{S_{3,1}, S_{3,2}, \ldots, S_{3, r-d}\right\}$,

where for $i=1, \ldots, r-d$,

$$
\begin{aligned}
S_{3, i}= & \left(X, X_{(t(i), i)}, X_{(t(i), j), s(1)}, X_{(t(i), i), s(1), s(2)}, \ldots,\right. \\
& X_{(t(i), j), s(1), s(2), \ldots, s(d)}, \\
& \left.X_{s(1), s(2), \ldots, s(d)}=Y\right) \mid \text { for } j=0, \ldots, m_{i}-1 \\
& \text { and } \left.j \neq x_{s(i)}\right\} .
\end{aligned}
$$

When $r=d$, the set $S_{3}$ is empty. For nonempty $S_{3}$, it contains $\sum_{i=1 \ldots r-d}\left(m_{t(i)}-1\right)$ paths whose length is $d+2$.

For example, let us consider $X=210$ and $Y=$ 201 in $G(4,3,2)$. If $(s(2), s(1))=(2,1)$, then

$$
\begin{aligned}
S_{1}= & \{(210,211,201),(210,200,201)\}, \\
S_{2}= & \{(210,220,221,201)\}, \\
s_{3}= & \{(210,010,011,001,201), \\
& (210,110,111,101,201), \\
& (210,310,311,301,201)\} .
\end{aligned}
$$


If $(s(2), s(1))=(1,2)$, then

$$
\begin{aligned}
S_{1}= & ((210,200,201),(210,211,201)\}, \\
S_{2}= & ((210,220,221,201)\} \\
S_{3}= & ((210,010,000,001,201) \\
& (210,110,100,101,201) \\
& (210,310,300,301,201)\}
\end{aligned}
$$

There are totally

$$
\begin{aligned}
d & +\sum_{i=1 \ldots r}\left(m_{s(i)}-2\right)+\sum_{i=1 \ldots r-d}\left(m_{t(i)}-1\right) \\
& =\sum_{i=1 \ldots r}\left(m_{i}-1\right)
\end{aligned}
$$

paths in $S_{1} \cup S_{2} \cup S_{3}$. The node-disjoint property is very clear. So, for each instance of $s(d), s(d-$ $1), \ldots, s(1)$, the paths contained in $S_{1} \cup S_{2} \cup S_{3}$ constitute a $C(X, Y)$ of width $\sum_{i=1 \ldots r}\left(m_{i}-1\right)$. The following lemma shows that the $C(X, Y)$ is the best.

Lemma 1. Suppose $X$ and $Y$ are two nodes in $G\left(m_{r}, m_{r-1}, \ldots, m_{1}\right)$ and they differ at $d$ digit positions denoted by $s(d), s(d-1), \ldots, s(1)$. Then, for each instance of $s(d), s(d-1), \ldots, s(1)$, the paths contained in $S_{1} \cup S_{2} \cup S_{3}$ constitute a $C^{*}(X, Y)$ of width $\sum_{i-1 \ldots,}\left(m_{i}-1\right)$.

Proof. According to the structure of $G\left(m_{r}\right.$, $\left.m_{r-1}, \ldots, m_{1}\right)$, a shortest path between $X$ and $Y$ must contain $d$ edges each of which equalizes a different digit. Hence, not more than $d$ node-disjoint shortest paths between $X$ and $Y$ exist. A second shortest path between $X$ and $Y$, whose length is $d+1$, has a form as shown in the set $S_{2, i}$. Each path in $S_{2, i}$ equalizes by two edges (the first and the last edges) the digit whose position is indicated by $s(i)$. There are at most $\sum_{i=1 \ldots d}\left(m_{s(i)}\right.$ $-2)$ node-disjoint second shortest paths between $X$ and $Y$. Since $\left|S_{1}\right|=d,\left|S_{2}\right|=\sum_{i=1 \ldots d}\left(m_{s(i)}-\right.$ 2 ), and each path in $S_{3}$ has a length $d+2$, the correctness of the lemma follows.

There are $d$ ! ways to specify $s(d), s(d-$ 1), $\ldots, s(1)$. Carefully observing the construction of $S_{1}$, we find that two different instances of $s(d), s(d-1), \ldots, s(1)$ will construct the same $S_{1}$ if and only if one can be obtained from the other by continuous cyclic rotations. Therefore, the following lemma holds.

Lemma 2. Suppose $X$ and $Y$ are two nodes in $G\left(m_{r}, m_{r-1}, \ldots, m_{1}\right)$ and they differ at $d$ digit positions. Then, $(d-1)$ ! different sets $S_{1}$ with respect to $X, Y$ can be constructed.

Suppose $a_{d}, a_{d-1}, \ldots, a_{1}$ and $b_{d}, b_{d-1}, \ldots, b_{1}$ are two different instances of $s(d), s(d-$ $1), \ldots, s(1)$. Without loss of generality, assume $a_{u}=b_{1}$ and $u<v$. The two sets $S_{2, u}$ and $S_{2, c}$ that are derived from the two instances, respectively, are identical if and only if $a_{k}=b_{k}$ for $1 \leqslant k \leqslant u$ -1 and $v+1 \leqslant k \leqslant d$, and $a_{k}=b_{k-1}$ for $u+1$ $\leqslant k \leqslant v$. But, under the latter condition, the two $S_{2, u}$, where $s(w) \neq a_{u}$ (and $b_{l}$ ), that are derived from the two instances, respectively, are different if $m_{w}>2$, or empty if $m_{w}=2$. This implies that under the assumption of $m_{i}>2$ for all $1 \leqslant i \leqslant r$, different instances of $s(d), s(d-1), \ldots, s(1)$ will construct different $S_{2}$. Therefore, we have the following lemma.

Lemma 3. Suppose $X$ and $Y$ are two nodes in $G\left(m_{r}, m_{r-1}, \ldots, m_{1}\right)$ and they differ at $d$ digit positions. If $m_{i}>2$ for all $1 \leqslant i \leqslant r$, then d! different $S_{2}$ with respect to $X, Y$ can be constructed.

It is easy to see that when $r>d$, each instance of $s(d), s(d-1), \ldots, s(1)$ will uniquely construct a set $S_{3}$. Therefore, we have the following lemma.

Lemma 4. Suppose $X$ and $Y$ are two nodes in $G\left(m_{r}, m_{r-1}, \ldots, m_{1}\right)$ and they differ at $d$ digit positions. If $r>d$, then $d$ ! different $S_{3}$ with respect to $X, Y$ can be constructed. If $r=d$, no such set can be constructed.

Combining Lemmas 1, 2, 3, and 4, we have the following theorem.

Theorem 5. Suppose $X$ and $Y$ are two nodes in $G\left(m_{r}, m_{r-1}, \ldots, m_{1}\right)$, where $m_{i}>2$ for all $1 \leqslant i$ $\leqslant r$, and they differ at $d$ digit positions. When $r>d, \quad(d-1) ! *(d !)^{2} C^{*}(X, Y) s$ of width $\sum_{i=1 \ldots r}\left(m_{i}-1\right)$ can be constructed. When $r=d$, 
$(d-1) ! * d ! C^{*}(X, Y) s$ of width $\sum_{i=1 \ldots r}\left(m_{i}-1\right)$ can be constructed.

The number of $C^{*}(X, Y)$ s in Theorem 5 represents a lower bound. More $C^{*}(X, Y) \mathrm{s}$ still exist.

The node connectivity of a graph is defined as the minimum number of nodes whose removal will disconnect the graph. We note that no container of width larger than $\sum_{i=1 \ldots r}\left(m_{i}-1\right)$ exists in $G\left(m_{r}, m_{r-1}, \ldots, m_{1}\right)$ because each node in $G\left(m_{r}, m_{r-1}, \ldots, m_{1}\right)$ has a degree $\sum_{i=1 \ldots r}\left(m_{i}-\right.$ 1). According to Menger's theorem [2], which states that the node connectivity of a graph is $\kappa$ if and only if there exist at least $\kappa$ node-disjoint paths between any two distinct nodes of the graph, we know that the node connectivity of $G\left(m_{r}, m_{r-1}, \ldots, m_{1}\right)$ is $\sum_{i=1 \ldots r}\left(m_{i}-1\right)$.

Corollary 6. The node connectivity of $G\left(m_{r}\right.$, $\left.m_{r-1}, \ldots, m_{1}\right)$ is $\sum_{i=1 \ldots r}\left(m_{i}-1\right)$.

\section{Wide diameter and fault diameter}

Let $d_{\kappa}(X, Y)$ denote the length of $C^{*}(X, Y)$ of width $\kappa$. That is, $d_{\kappa}(X, Y)=\min (l(C(X, Y)) \mid$ for all $C(X, Y)$ s with $w(C(X, Y))=\kappa$ ). The $\kappa$ wide diameter [5] of $G\left(m_{r}, m_{r-1}, \ldots, m_{1}\right)$, which is denoted by $d_{\kappa}\left(G\left(m_{r}, m_{r-1}, \ldots, m_{1}\right)\right)$, is defined as the maximum of $d_{\kappa}(X, Y)$ s for all pairs of nodes $X, Y$ in $G\left(m_{r}, m_{r-1}, \ldots, m_{1}\right)$. That is, $d_{\kappa}\left(G\left(m_{r}, m_{r-1}, \ldots, m_{1}\right)\right)=\max \left\{d_{\kappa}(X, Y) \mid\right.$ for all pairs of nodes $X, Y$ in $G\left(m_{r}, m_{r-1}, \ldots, m_{1}\right)$ \}.

Let $\kappa=\sum_{i=1 \ldots r}\left(m_{i}-1\right)$ denote the connectivity of $G\left(m_{r}, m_{r-1}, \ldots, m_{1}\right)$. According to the discussion of Section 2, we know that for any two nodes $X, Y$ in $G\left(m_{r}, m_{r-1}, \ldots, m_{1}\right), d_{\kappa}(X, Y)=$ $d+2$ if $d<r$, and $d+1$ if $d=r$. Thus, $d_{k}(X, Y)$ is maximized as $d=r$ or $d=r-1$, and $d_{\kappa}\left(G\left(m_{r}, m_{r-1}, \ldots, m_{1}\right)\right)$ is computed as follows.

Theorem 7. $d_{\kappa}\left(G\left(m_{r}, m_{r-1}, m_{1}\right)\right)=r+1$.

The fault diameter [6] of $G\left(m_{r}, m_{r-1}, \ldots, m_{1}\right)$ is defined as its maximum diameter after at most $\kappa-1$ nodes and their incident edges are removed. By definition, $d_{\kappa}\left(G\left(m_{r}, m_{r-1}, \ldots, m_{1}\right)\right)$ is an upper bound on the fault diameter of $G\left(m_{r}, m_{r-1}, \ldots, m_{1}\right)$. Let $X=x_{r} x_{r-1} \ldots x_{1}$ and $Y=y_{r} y_{r-1} \ldots y_{1}$ be two nodes in $G\left(m_{r}, m_{r-1}\right.$, $\left.\ldots, m_{1}\right)$, where $x_{i} \neq y_{i}$ for $1 \leqslant i \leqslant d$ and $x_{j}=y_{j}$ for $d+1 \leqslant j \leqslant r$ such that $d_{\kappa}(X, Y)$ has maximum value. When $d=r$, if we remove the $r$ nodes: $x_{r} x_{r-1} \ldots x_{2} y_{1}, \quad x_{r} x_{r-1} \ldots x_{3} y_{2} x_{1}, \ldots$, $y_{r} x_{r-1} \ldots x_{1}$, then the distance between $X$ and $Y$ will become $r+1$. Similarly, when $d=r-1$, the distance between $X$ and $Y$ will become $r+1$ after removing the $r-1$ nodes: $x_{r} x_{r-1} \ldots x_{2} y_{1}$, $x_{r} x_{r-1} \ldots x_{3} y_{2} x_{1}, \ldots, x_{r} y_{r-1} x_{r-2} \ldots x_{1}$. This gives a lower bound of $r+1$ on the fault diameter of $G\left(m_{r}, m_{r-1}, \ldots, m_{1}\right)$. Hence, the following theorem holds.

Theorem 8. The fault diameter of $G\left(m_{r}\right.$, $\left.m_{r-1}, \ldots, m_{1}\right)$ is $r+1$.

In [8], Tien and Raghavendra showed that the diameter of a faulty $n$-dimensional hypercube with at most $2 n-3$ faults is bounded above by $n+2$, provided the source node and the destination node are not isolated. This bound can be further reduced to $n+1$ or $n$ if extra conditions are satisfied.

\section{Remarks}

A method to construct ontainers with maximum width for the hypercube graph has been proposed in [7]. The generalized hypercube graph is a generalization of the hypercube graph. In this paper, besides containers with maximum width, we constructed the best containers and computed the wide diameter and the fault diameter for the generalized hypercube graph. It is of both theoretical interest and practical importance to solve the container problem. The existence of containers can increase transmission rate as well as transmission reliability. For a network having a container of width $\kappa$ between every two nodes, a transmission path between the source node and the destination node can be guaranteed even if $\kappa-1$ node faults have occurred. The fault diameter, on the other hand, estimates the maximum length of the transmission path between the 
source node and the destination node under the situation of at most $\kappa-1$ node faults.

The $k$-ary $n$-cube graph [4] is another generalization of the hypercube graph. It differs from the generalized hypercube in two: (1) each dimension has the same width: (2) the nodes belonging to the same dimension are connected as a ring (they are connected as a clique for the generalized hypercube graph). The interested readers are encouraged to determine containers and the fault diameter of the $k$-ary $n$-cube graph.

\section{References}

[1] L.N. Bhuyan and D.P. Agrawal, Generalized hypercube and hyperbus structures for a computer network, IEEE Trans. Comput. 33 (1984) 323-333.
[2] F. Buckley and F. Harary, Distance in Graphs (AddisonWesley, Reading, MA, 1990).

[3] G.H. Chen and D.R. Duh, Topological properties, communication, and computation on WK-recursive networks, Networks 24 (1994) 303-317.

[4] W.J. Dally, Performance analysis of $k$-ary $n$-cube interconnection networks, IEEE Trans. Comput. 39 (1990) $775-785$.

[5] D.F. Hsu, On container width and length in graphs, groups, and networks, IEICE Trans. Fundamentals E77-A (1994) $668-680$.

[6] M.S. Krishnamoorthy and B. Krishnamurthy, Fault diameter of interconnection networks, Comput. Math. Appl. 13 (1987) 577-582.

[7] Y. Saad and M.H. Schultz, Topological properties of hypercubes, IEEE Truns. Comput. 37 (1988) 867-872.

[8] S.B. Tien and C.S. Raghavendra, Algorithms and bounds for shortest paths and diameter in faulty hypercubes, IEEE Trans. Parallel Distributed Systems 4 (1993) 713-718. 